\section{Mitochondrial base prediction}

SIR - Goto et al. reported ${ }^{1}$ that a heteroplasmic mutation in the mitochondial transfer RNA $^{\text {leu(UUR) }}$ gene is found in familial cases of the MELAS subgroup of mitochondrial encephalomyopathies. This mutation replaces a conserved A residue of the DHU loop of the tRNA with a G, and the authors suggest that this alteration to the structure of the rRNA may play a role in pathogenesis. Although this idea is perfectly plausible, the affected nucleotide pair has an additional, identified function, suggesting an alternative pathogenic mechanism.

The tRNA ${ }^{\text {leu(UUR) }}$ gene of vertebrate mitochondrial DNA overlaps a transcriptional attenuator, functionally identified in vitro in human cells ${ }^{2,3}$ and highly conserved at the sequence level (11/13 nucleotides) in mouse, rat, cow, frog ${ }^{4}$ and chicken. This sequence functions bidirectionally ${ }^{5}$, and its action seems to be mediated by specific protein-binding ${ }^{2,3}$. This is believed to be the principal mechanism regulating the relative synthetic rates of mitochondrial, ribosomal, messenger and transfer RNA in vertebrate cells, because the rRNA genes, plus those encoding tRNA ${ }^{\text {phe }}$ and tRNA ${ }^{\text {val }}$, are found, in the transcription unit of the heavy strand, upstream of the attenuator. This is supported by observations that these two rRNAs are synthesized at much high levels than the remainder ${ }^{2}$.

We suggest that a mutation in the attenuator could cause disease by an altered affinity for the protein factor(s) involved in termination, thus leading either to an excess or a deficiency in the rate of synthesis of diffeent classes of RNA. This hypothesis has a clear and easily testable prediction, namely that the protein-binding characteristics of the attenuator, as well as the efficiency of transcriptional termination in vitro, should be altered by this base change.

It might also be expected that some mutations in the nuclear gene encoding the termination factor could have a similar effect on its binding affinity or specificity, resulting in symptoms indistinguishable from MELAS. Alternatively, other alleles of this gene could regulate penetrance by acting as suppressors of the mitochondrial mutation.

Abdellatif El Meziane HOWARD T. JACOBS

Institute of Genetics,

University of Glasgow,

Church St,

Glasgow G11 5JS, UK

1. Goto, Y., Nonaka, I. \& Horal, S. Nature 348, 651-653 (1990).

Christianson, T. W. \& Clayton, D. A. Molec. cell. Biol. 8 4502-4509 (1988).

3. Kruse, B., Narasimham, N. \& Attardi, G. Ce//58, 391-397 (1989).

4. Ei Meziane. A., Callen, J.-C. \& Mounolou, J.-C. EMBO $/ 8$ 1649-1655 (1989)

5. Christianson, T. W. \& Clayton, D. A. Proc. natn. Acad. Sci. U.S.A. 83, 6277-6281(1986).

\section{Clues to action of cdc25 protein}

SIR - The cdc25 protein is required to promote dephosphorylation of a specific tyrosine residue Tyr 15 in $\mathrm{p} 34^{\mathrm{cdc} 2}$, which leads to activation of the $\mathrm{p} 34^{\mathrm{cdc} 2} /$ cyclin B kinase and consequently to the onset of mitosis $^{1-3}$. Comparisons between the aminoacid sequences of cdc 25 and of previously published protein tyrosine phosphatases (PTPases) have not revealed extensive phate acceptor in the enzyme mechanism $)^{3,4}$. Such a stoichiometric mode of action might explain the dosage effects of cdc 25 over onset of mitosis.

In vertebrate cells, dephosphorylation of both Thr14 and Tyr 15 in $\mathrm{p} 34^{\mathrm{cdc} 2}$ is required for the activation of the kinase (C. J. Norbury, J. J. Blow and P. N, manuscript submitted). It is therefore of interest that the cdc25 protein is similar in sequence to a protein phosphatase with activity against both tyrosine and serine (and thus probably threonine) phosphate residues. If the cdc 25

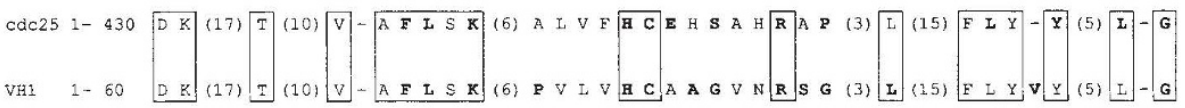

Sequence comparison between Schizosaccharomyces pombe cdc25 protein and vaccinia virus $\mathrm{VH} 1$ protein phosphatase. Identical matches are boxed. Amino acids represented in bold in the cdc25 sequence are conserved among cdc25 homologues, whereas those in the VH1 sequence are conserved in protein tyrosine phosphatases. The line marks the 'signature sequence' containing the putative catalytic site.

similarities, and so it has been unclear whether cdc 25 acts directly to dephosphorylate $\mathrm{p} 34^{\mathrm{cdc} 2}$. But we have found that the cdc 25 protein shares a degree of similarity with a newly identified phosphatase which can dephosphorylate both serine and tyrosine residues ${ }^{4}$. This is the vaccinia virus protein $\mathrm{VH} 1$, which gives the highest score in the PIR protein database, after a search for similarities in sequence to the conserved amino acids among cdc 25 homologues from yeast to human. A region of 92 amino acids in the $C$ terminus of cdc 25 shows 21 per cent identity to VH1 (see figure). This region includes a motif, HC (5) R, which is found in all PTPases and is thought to include the putative catalytic site. These cysteine (C) and arginine $(\mathrm{R})$ residues are absolutely essential for phosphatase activity ${ }^{4,5}$.

The possibility that the cdc 25 protein might act directly to dephosphorylate Tyr 15 in $\mathrm{p} 34^{\mathrm{cdc} 2}$ gains further support from recent genetic and biochemical work investigating the role of $\mathrm{cdc} 25$. Fission yeast cells that are unable to divide because they contain a mutation in or are deleted for $c d c 25^{+}$can be rescued by overexpressing a $\mathrm{T}$-cell tyrosine phosphatase $^{1}$. Also, purified, bacterially produced string protein (a Drosophila cdc25 homologue) can dephosphorylate partially purified Xenopus p34 $4^{\text {cdc2 }} /$ cyclin (ref 2); similar results are obtained using bacterially produced human CDC25 and highly purified $\mathrm{p} 34^{\mathrm{cdc} 2} /$ cyclin obtained from starfish oocytes (ref. 3 on page 242 of this issue). In all cases in vitro dephosphorylation of $\mathrm{p} 34^{\mathrm{cdc} 2}$ results in an increase in kinase activity. The similarities in sequence between cdc 25 and the VH 1 tyrosine/serine phosphatase obviously suggest that the cdc 25 protein acts directly as a phosphatase. But until further enzymatic studies are performed it cannot be ruled out that cdc 25 functions in another manner. For example, it could act noncatalytically, leading to irreversible phosphate transfer to the cysteine residue (thought to be the nucleophilic phos- protein has activity against both tyrosine and threonine phosphate residues, then it could dephosphorylate both $\mathrm{Thr} 14$ and Tyr15 bringing about onset of mitosis in vertebrate cells.

Sergio Moreno Paul Nurse

ICRF Cell Cycle Group,

Microbiology Unit,

Department of Biochemistry,

Oxford University,

Oxford OX1 3QU, UK

1. Gould K. L., Moreno, S., Tonks, N. K. \& Nurse, P. Science 250, 1573-1576 (1990)

2. Kumagai, A. \& Dunphy, W. G. Cell 64, 903-914(1991). 3. Strausfeld, U. et al.. Nature, 351, 242-245 (1991).

4. Guan, K., Broyles, S. S. \& Dixon, J. E. Nature 350 , 359-362 (1991)

5. Streuli, M., Kreuger, N. X., Thai, T., Tang, M. \& Saito, H. EMBO \&. 9, 2399-2407 (1990).

\section{Conscious or unconscious?}

SIR - Goodale et al. ${ }^{1}$ may have confused an important distinction relating to conscious and unconscious functions. They state that their evidence suggests that "... the visual processing underlying conscious perceptual judgements must operate separately from that underlying the 'automatic' visuomotor guidance of skilled actions ...". This implies a separation between processing pathways for conscious and unconscious functions generally.

Perhaps Goodale et al. based such a separation on the assumption that all the perceptual tests in which their subject failed represented tests of conscious awareness of the signal. But we have recently gained evidence for the possibility that such tests might be performed successfully by a normal subject even without awareness ${ }^{2}$. Given electrical stimuli in the somatosensory (ventrobasal) thalamus, human subjects correctly detected the presence of a stimulus (in forced choice responses), even when they were completely 